\title{
Neocortical rhythm entrainment by parvalbumin-positive interneurons across cortical layers
}

François David ${ }^{1}$, Mélodie Borel ${ }^{1}$, Suleman Ayub² ${ }^{2}$ Patrick Ruther ${ }^{2}$, Luc J. Gentet ${ }^{1}$

\author{
1) Lyon Neuroscience Research Center, INSERM U1028, UMR5292, UCBL. Centre \\ Hospitalier Le Vinatier - Bâtiment 462 - Neurocampus, 95 boulevard Pinel, 69675 Bron \\ Cedex, France \\ 2) University of Freiburg, Department of Microsystems Engineering (IMTEK), Freiburg, \\ Germany BrainLinks-BrainTools, University of Freiburg, Germany
}

\begin{abstract}
Neocortical interneurons provide local inhibition responsible for organizing neuronal activity into brain oscillations that subserve several functions such as memory, attention and neuronal communication. However, little is known about the contribution of interneurons to the entrainment of neocortical oscillations across cortical layers. Here, using layer-specific optogenetic stimulations with micro-Light-Emitting-Diode ( $\mu$ LED) arrays, directed toward parvalbuminexpressing (PV) interneurons in non-anesthetized awake mice, we find that supragranular layer stimulations of PV neurons were most efficient at entraining supragranular layer neurons and local field potential (LFP) oscillations at gamma frequencies $(\gamma: 25-80 \mathrm{~Hz})$, whereas infragranular layer stimulation of PV neurons better entrained delta $(\delta: 2-5 \mathrm{~Hz})$ and theta $(\theta: 6-10 \mathrm{~Hz})$ frequency LFP oscillations. We found that PV neurons tightly control the transmission of multiple rhythms to the network across cortical layers in an orientation-selective manner. Intrinsic resonant properties of neurons could underlie such layer-specific properties of rhythm entrainment.
\end{abstract}




\section{Introduction}

Neocortical brain rhythms are emergent neuronal circuit properties that underlie many functional mechanisms, such as plasticity (Chauvette et al., 2012; Durkin et al., 2017; Peyrache and Seibt, 2020), synaptic integration (Wehr and Zador, 2003) and inter-area communication (Fries, 2015). Locally, they manifest themselves as electrophysiological bouts of oscillations at frequencies from $0.05 \mathrm{~Hz}$ to $500 \mathrm{~Hz}$, visible at the EEG level. Their emergence and maintenance both depend on tight interactions between excitatory and inhibitory inputs (Tiesinga and Sejnowski, 2009) and intrinsic neuronal properties (Wang, 2010).

Among the different subclasses of cortical GABAergic interneurons, parvalbumin-containing (PV) cells and fast-spiker (FS) neurons have been shown to play a critical role in the emergence, maintenance and termination of sleep slow waves (Shu et al., 2003; Zucca et al., 2019) and in the pacemaker activity of $\gamma$ oscillations (Cardin et al., 2009; Sohal et al., 2009; Kuki et al., 2012; Welle and Contreras, 2016) through the strong and rapid inhibition they provide onto the soma and proximal dendrites of pyramidal neurons (Gentet et al., 2012; Tremblay et al., 2016). Indeed, their neuronal activity has been shown to be correlated with various brain rhythms throughout the cortex (Klausberger et al., 2005; Peyrache and Destexhe, 2019). Therefore, cortical FS cells, which mainly consist of GABAergic PV interneurons (Hu et al., 2014) play a major role in controlling the excitability of the network (Ferguson and Gao, 2018) through their widespread connections to local pyramidal neurons (Packer and Yuste, 2011).

Brain oscillations modulate both bottom-up and top-down cognitive processes (Arnal and Giraud, 2012; van Kerkoerle et al., 2014; Bastos et al., 2015) through the layer-based functional architecture of the neocortex. For example, somatosensory inputs from the whiskers mainly enter the cortical column at the level of granular layer 4, while contextual inputs are carried into supragranular layers 1-3 (Douglas and Martin, 2004; Phillips et al., 2016) and ascending inputs controlling the input-output function of the column into infragranular layers 5-6 (Ahissar and Staiger, 2010). How the various PV cells that are present in both supra- and infragranular layers entrain and transfer different brain rhythms within a cortical column remains to be determined.

Here, we investigated the differential contribution of infra- versus supra-granular layer PV interneurons to the amplification of the oscillatory regimes of the mouse primary somatosensory 
barrel cortex (S1) via rhythmic and local activation of local PV-containing interneurons with arrays of miniaturized sources of light ( $\mu$ LEDs - see Ayub et al., 2017, 2020). In non-anaesthetized headfixed mice during quiet wakefulness in which spontaneous activity covers a large range of spontaneous oscillation frequencies $(0.5$ - $100 \mathrm{~Hz}$, Buzsáki and Draguhn, 2004). Using a combination of virally-mediated cre-dependent channelrhodopsin expression in transgenic PV-cre mice (Madisen et al., 2012) combined with neuronal ensemble recordings, we were able to optogenetically activate PV neurons located in either supra- or infragranular layers of S1 and observe their impact at the level of local field potentials (LFP) and cortical neuronal activity. We uncovered stronger impact of infragranular-layer PV activation at $\delta$ and $\theta$ frequencies on LFPs throughout the column, while supragranular PV cell activation better entrained oscillations at $\gamma$ rhythms $(25-80 \mathrm{~Hz})$. Furthermore, we found that supragranular cortical neurons were better entrained than their infragranular counterparts at $\gamma$ frequencies, but also surprisingly at both $\delta$ and $\theta$ frequencies. Our results reveal how cortical PV neurons can differentially impact neocortical rhythms based on their anatomical location across layers.

\section{Material and Methods}

\section{Transgenic mice}

All animal experiments were conducted after approval by the local ethical committee of the University of Lyon and the Ministry of Science (Protocol number: Apafis \#4613) in accordance with the European directive 86/609/EEC on the protection of animals used for experimental and other scientific purposes. We used offsprings of PV-Cre driver mice (008069, Jackson). All animals used in this study were group-housed in the vivarium under normal light cycle conditions.

\section{Surgery and recording}

All surgical procedures were performed under 1.5-2\% isoflurane anaesthesia, after prior injection of $5 \mathrm{mg} / \mathrm{kg}$ carprofen. First, animals (6-12 wk old) were implanted with a lightweight head-bar and a recording chamber. A small craniotomy was performed $300 \mu \mathrm{m}$ lateral to the $\mathrm{C} 2$ barrel column (identified through intrinsic optical imaging) and adeno-associated virus (AAV1.EF1a.DIO.hChR2(H134R)-eYFP.WPRE.hGH; Penn Vector Core, University of 
Pennsylvania) was injected into the $\mathrm{C} 2$ barrel column at depths of $250 \mu \mathrm{m}, 500 \mu \mathrm{m}, 750 \mu \mathrm{m}$ and $1000 \mu \mathrm{m}$ (100 $\mathrm{nl}$ per injection site). Mice were then progressively habituated to head-fixed conditions with regular rewards for 10 days before recording. On the day of recording, the craniotomy at the site of viral injection was enlarged, a second small craniotomy was performed above the C2 column and the dura was removed at both locations $300 \mu \mathrm{m}$ apart in anticipation for the recording electrode and $\mu$ LED array insertions. Animals were allowed to recover from anaesthesia for at least $2 \mathrm{~h}$, during which the craniotomy was kept humidified with artificial cerebrospinal fluid (ACSF). The $\mu$ LED array and a linear 32-channel silicon electrode were slowly lowered into the brain, with the light sources from the $\mu \mathrm{LED}$ array facing the $\mathrm{C} 2$ barrel column (Figure 1a). The electrode and the $\mu$ LED array were fixed together with a small amount of silicon gel (Kwik-Cast) for higher mechanical stability. Signals were acquired full-band and continuously using a Multi-Electrode-Array portable 32-channel (ME32-FAI-mPA, Multichannel Systems MCS GmbH) at $20 \mathrm{kHz}$. Optogenetic stimulation time points were simultaneously recorded on an extra-digital channel.

\section{Optogenetic stimulation}

We used two sets $\mu$ LED array types (Ayub et al., 2020) that differed only by their inter- $\mu$ LED distances ( $\mu$ LED pitch values of 150 or $250 \mu \mathrm{m}$ ). Individual $\mu$ LEDs were controlled using a custom-made Arduino-based driver. Light intensity was set to $3 \mu \mathrm{W}\left(\mathrm{E}=0.4 \mathrm{~mW} . \mathrm{mm}^{-2}\right.$ ) which was previously found to be sufficient to activate $\mu \mathrm{LED}$-specific subsets of constitutivelyexpressing ChR2 PV neurons on the densest array (Ayub et al., 2020). Trains of 10 light pulses interspaced by 4 seconds (Figure 1a) mimicked the transient aspects of brain oscillations which often occur in short bursts. The stimulation frequencies were chosen to cover a wide range of physiological brain rhythms (i.e. $0.5-100 \mathrm{~Hz}$ : Slow waves $(0.5-1 \mathrm{~Hz})$, Delta $(\delta, 2-5 \mathrm{~Hz})$, Theta $(\theta$, 6-10 Hz), Beta $(\beta, 20-25 \mathrm{~Hz})$ and $\operatorname{Gamma}(\gamma, 25-80 \mathrm{~Hz}))$. Each stimulation frequency-LED combination was repeated 6 to 10 times per experiment in a randomized order. 


\section{Analysis}

Local field potential (LFP) was derived from the raw signal downsampled at $1 \mathrm{kHz}$. A recording site within the granular layer was used as common local reference site for supra- and infra-granular differential LFP measurements (Herreras, 2016) and therefore subtracted from the infra and supra LFP recorded traces. The analysis was also run on unipolar LFP signals with the reference site in the recording chamber, which qualitatively led to similar results. To measure the changes evoked by each stimulation frequency, we performed a time-frequency analysis restricted to that particular frequency of all corresponding LFPs using short-time Fourier transforms. The LFP modulation index was the ratio of this power measure and a control value obtained from the concatenated signal outside the stimulation intervals for a number of samples identical to the number of trials randomly pooled. This control value was then computed 60 times to avoid sample size variability during the control period and then averaged to get the control value. The LFP modulation index was then derived from the relative change of power during the light stimulation compared to the one outside the stimulation (an index of 0 indicates no change and 1 indicates a 2 -fold increase).

Single- and multi- unit neuronal activity was extracted from the high-pass filtered signal and sorted using Klusta suite software (https://klusta.readthedocs.io/, (Rossant et al., 2016)). Neuronal clusters were categorized either as belonging to fast-spiking (FS) neurons if their half-width durations were smaller than 0.6 - 0.75 times the trough-to-peak interval time or to putative regularspiking (RS) neurons for the others based on their spike-shape (Barthó et al., 2004). Since we measured the proportional changes in neuronal firing rates induced by optogenetic stimulations, neuronal clusters with a firing rate below $0.1 \mathrm{~Hz}$ were discarded from the analysis. Units were further categorized with respect to their response to at least one $\mu$ LED stimulation into "positively responding' if their firing rates more than doubled (modulation index greater than 1) or 'negatively responding' if the firing rates decreased by more than $20 \%$. Units that did not fall within these criteria were discarded. The neuronal firing modulation index was defined as the ratio of the firing rate observed during the light stimulation and the firing rate measured in-between light pulses. This measure was designed to quantify how the neuronal firing increased or decreased synchronously with the stimulation. The neuronal coherence was estimated as the module of the spike phase histograms relative to the optogenetic stimulation and was intended to measure the strength of entrainment per neuron. For representation purposes, neuronal firing modulation curves 
(representing the neuronal response as a function of stimulation frequency) were smoothed using a 3 points window moving average. Neurons located above $-400 \mu \mathrm{m}$ or below $-500 \mu \mathrm{m}$ were defined as belonging to the supra- or the infra-granular layers respectively (Lefort et al., 2009). Our results were maintained when we both shifted or extended the granular layer position by 100 $\mu \mathrm{m}$ beneath its lower boundary of $-500 \mu \mathrm{m}$.

\section{Statistics}

Modulation indexes were tested based on non-parametric paired or unpaired (Wilcoxon signed or unsigned, respectively) tests. Phase distributions were tested for non-uniformity with the Rayleigh test and phase distribution pairs were compared with the Kuiper test. We reported significant value when p-values $<0.05$. Results are reported as means \pm standard error of the mean (SEM), which is extended in the circular domain as the circular mean and the circular deviation over the square root of the sample size. The distribution modality of modulation index relative to stimulation frequency was assessed using a Hartigan dip test (Hartigan and Hartigan, 1985).

\section{Results}

Local and global inhibition by PV neurons in the somatosensory cortex

We explored how a rhythmic and specific activation of PV neurons impacted the neuronal activity in the mouse primary somatosensory cortex (S1) based on its depth and frequency (Figure 1a). Spike shape analysis of 485 neuronal clusters $(n=7$ mice $)$ indicated that the majority of positively responding neurons (22 out of 27, Figure 1 b,d,e) were classified as fast-spiking neurons (FS), and were therefore categorized as putative PV cells based on their action potential waveforms and responses to light stimulation (Kawaguchi and Kubota, 1997). Furthermore, the maximal action potential (AP) response was always observed when the $\mu$ LED facing the neuron was turned on (Figure 2a). Therefore, light illumination was spatially-constrained to cortical depths directly facing or neighboring the stimulating $\mu \mathrm{LED}$, confirming our previous results (Ayub et al., 2020). 
a
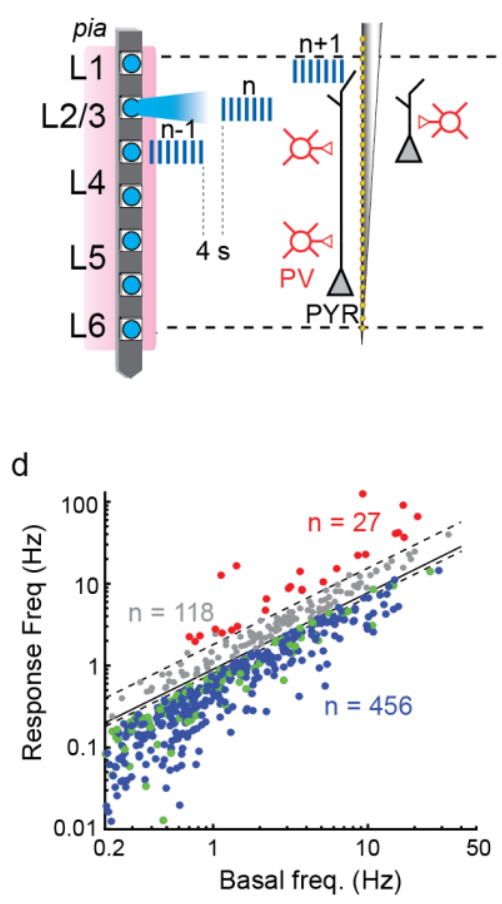

b

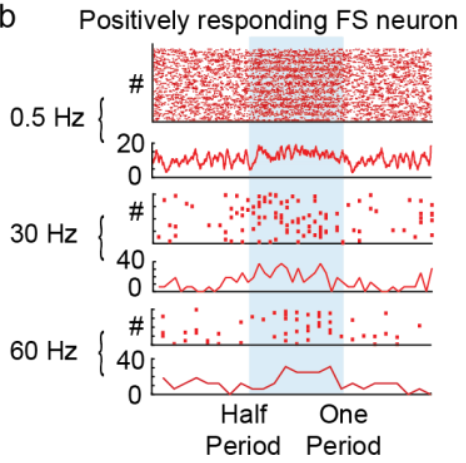

C Negatively responding RS neuron
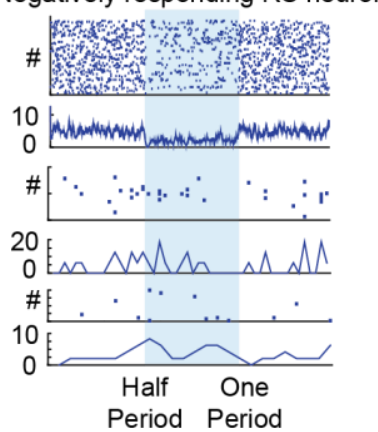

e
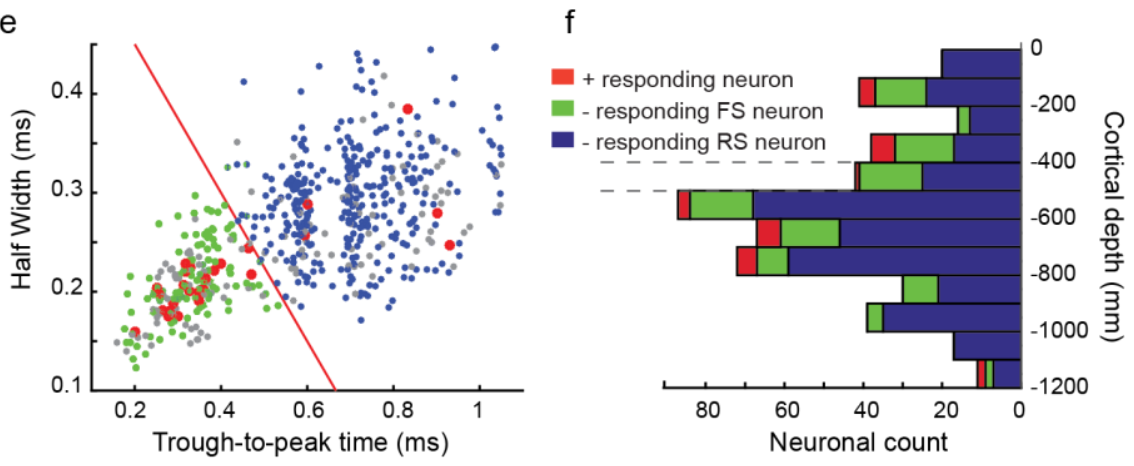

Figure 1: Parvalbumin-positive neurons control of inhibition in the cortical column. a)

Schematic positioning of the $\mu$ LED array and multi-site electrode facing each other across cortical layers (L1-6) of mouse barrel cortex S1. PY: pyramidal neurons, PV: parvalbumin positive interneurons. $\mu$ LED light pulses are sequentially emitted every 4 seconds. b) Raster plot and PSTH for a positively-responding FS neuronal unit at $0.5,30$ and $60 \mathrm{~Hz}$. Blue shaded area: $\mu \mathrm{LED}$ 'ON' time. $\mathrm{X}$-axis scale is adjusted to the period of the rhythmic stimulation. c) Same as b for a negatively responding RS unit. d) Firing frequency response (y-axis) for positively responding FS neurons (red), negatively-responding neurons (RS blue and FS green), and non-responding neurons (grey) compared to their basal firing rate (x-axis). Diagonal dashed lines top and bottom limits of non-responding neurons (dashed), and the continuous line is the identity line. e) Spike shape properties of positively-responding neurons (red) and negatively responding RS (blue) and FS (green) neurons and non-responding neurons (grey). f) Neuronal depths distribution of positively- and negatively-responding FS and RS neurons ( $\mathrm{n}=7$ mice). The dashed lines indicate the upper and lower limits of the granular.

The remaining population of recorded neurons displayed either decreased firing of at least $20 \%$ (Figure 1c-d, $\mathrm{n}=456$ neurons) or no significant change in their spiking rate in response to light pulses ( $\mathrm{n}=103$ neurons). Amongst the inhibited neuronal clusters, a majority (352 out of 456) was 
classified as regular-spiking (RS) neurons based on their spike-shape (Figure 1e). Interestingly however, a large proportion of FS neurons, also responded with decreased firing rates (104 out of 149 neurons) suggesting an inhibition of some FS neurons by PV neurons. Recorded neurons spread all along the cortical depth (Figure 1f). In contrast with the positively responding neurons, the decrease in firing of the negatively responding neurons spread well beyond the cortical depth facing the stimulating $\mu$ LED (Figure 2a,b). The spatial extent of inhibition in the cortical column, suggests either axonal conduction by GABAergic neurons and/or a dendritic integration of the inhibition across multiple layers by pyramidal neurons.

Since the specific activation of PV neurons had both excitatory and inhibitory effects on cortical network activity, each with different temporal dynamics (Figure 2a, b), we tested how light stimulations at frequencies spanning from 0.5 to $100 \mathrm{~Hz}$ differentially modulated responding neurons and the network global activity. While positively responding neurons remained rhythmically entrained at nearly all stimulation frequencies (Figure 2c), neuronal firing modulation appeared reduced for higher frequencies (above $40 \mathrm{~Hz}$ ) compared to lower frequencies (Figure 2f). In parallel, phasic inhibition weakened with stimulation frequencies above $20 \mathrm{~Hz}$ (Figure 2d) and became mostly insignificant (except at low $\gamma$ frequencies $(50-60 \mathrm{~Hz}$; Figure 2f)), indicating the potential existence of a cut-off frequency in the ability of local PV neurons to rhythmically entrain the neocortical network overall activity.

Interestingly, the stimulation frequency also affected the spatial extent of the modulation. Optogenetic stimulations were $\mu$ LED-specific for positively responding neurons at low frequencies (for example at $0.5 \mathrm{~Hz}$, red curve in Figure 2e; Facing $\mu \mathrm{LED}$ modulation index $=2.00$ $\pm 0.95, \mathrm{n}=18, \mathrm{p}=0.003 ; 400 \mu \mathrm{m}$ apart: modulation index $=0.26 \pm 0.12, \mathrm{n}=14, \mathrm{p}=0.11$; nonzero mean, Wilcoxon rank sum test). However, at faster light stimulation (60 Hz, yellow curve in Figure 2e the firing of positively responding neurons up to $600 \mu \mathrm{m}$ apart still increased (Facing $\mu \mathrm{LED}$ : modulation index $=1.41 \pm 0.33, \mathrm{n}=16, \mathrm{p}=0.0009 ;-600 \mu \mathrm{m}$ apart: modulation index $=$ $0.46 \pm 0.68, \mathrm{n}=13, \mathrm{p}=0.005$; non-zero mean Wilcoxon rank sum test).

In contrast, the rhythmic inhibition remained significant for a large depth range at all frequencies (Figure $2 b, e$ ), while remaining maximal when the light was delivered from the facing $\mu$ LED compared to neighbouring $\mu \mathrm{LEDs}$ (for instance at $0.5 \mathrm{~Hz}$ stimulation frequency, Figure $2 \mathrm{e}$; facing 
$\mu \mathrm{LED}$ modulation index $=-0.58 \pm 0.02, \mathrm{n}=184 \mathrm{vs} 400 \mu \mathrm{m}$ apart, modulation index $=-0.53 \pm 0.02$, $\mathrm{n}=169, \mathrm{p}=0.006)$. Partly consistent with the cut-off frequency described above for the positivelyresponding responses, the phasic inhibition became weaker at higher frequencies (Figure 2e, modulation index at $60 \mathrm{~Hz}=-0.17 \pm 0.11, \mathrm{p}=0.002, \mathrm{n}=98$ ) and less layer-specific (Figure 2e). This suggests that higher frequencies are more prone to equal spreading of rhythmic inhibition throughout layers, albeit at a weaker intensity.

a

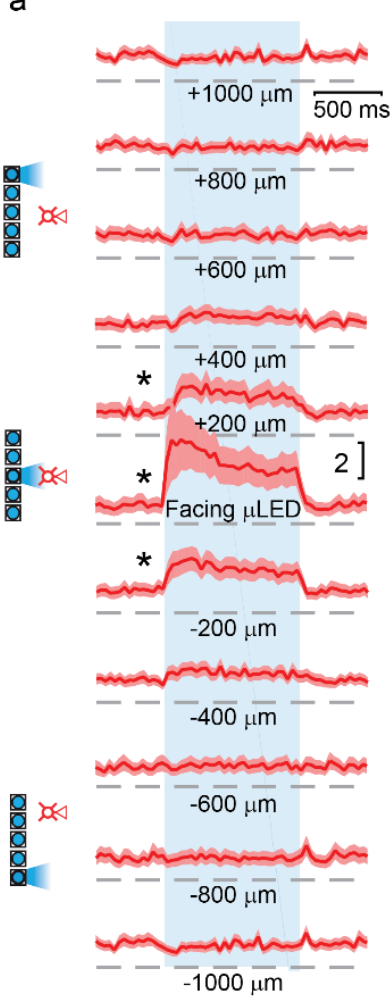

b

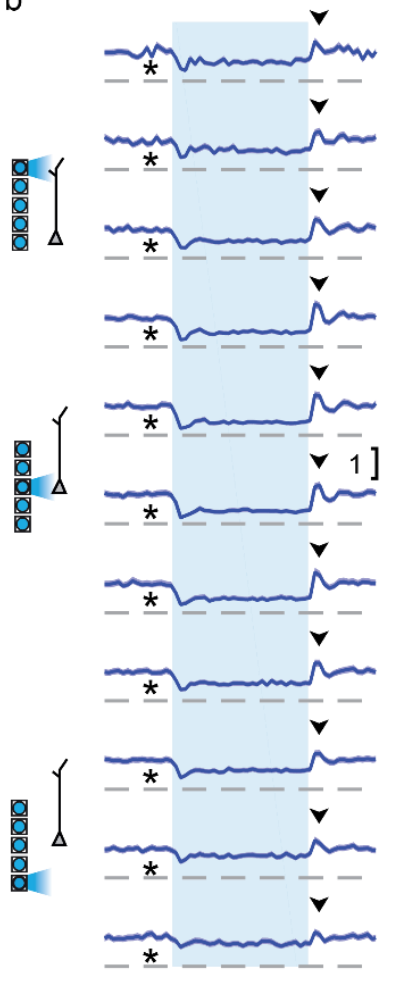

c

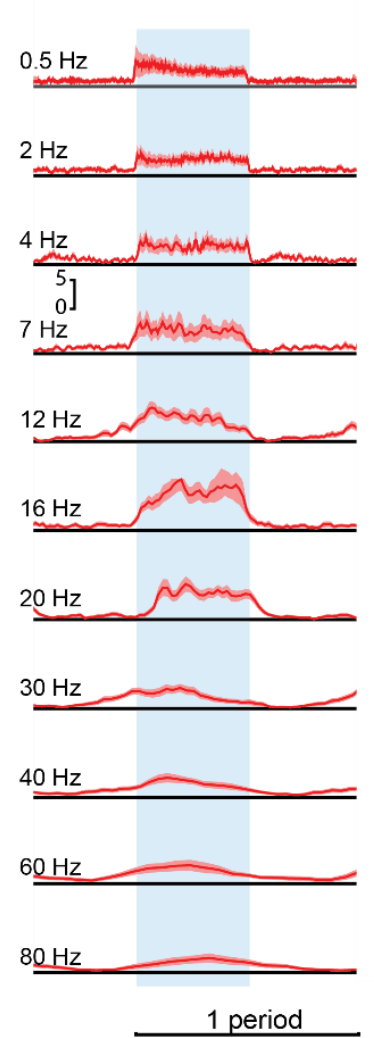

1 period d
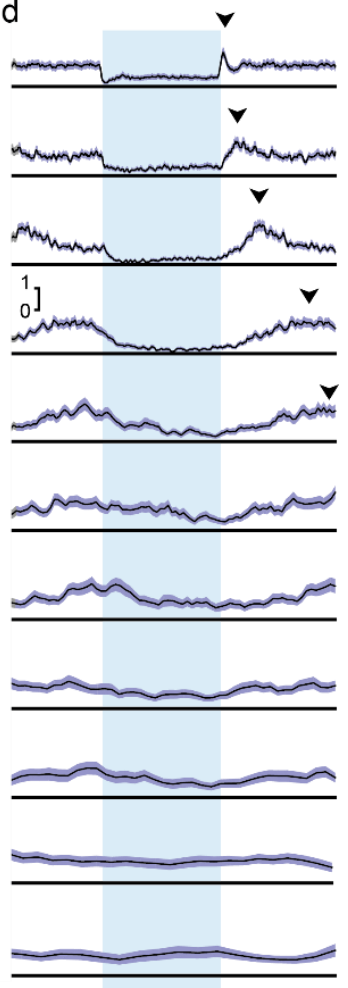

e

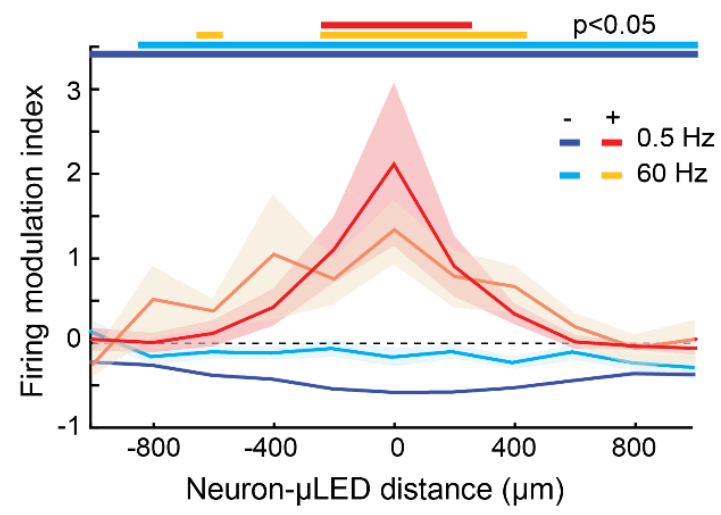

Figure 2: Frequency-dependent spread of phasic inhibition a) Average normalized response at $0.5 \mathrm{~Hz}$ stimulation ( \pm SEM (shaded area)) of positively-responding neurons based on neuronal 
location relative to the $\mu \mathrm{LED}$ 'ON' (blue rectangle). * indicates p-value $<0.05$, Wilcoxon signedrank test ( $8 \leq \mathrm{n} \leq 18$ neurons per distance to the $\mu \mathrm{LED}$ ) for difference relative to $\mu$ LED 'OFF' times. b) Same for negatively responding neurons. $32<\mathrm{n}<184$ neurons per distance to the $\mu$ LED. Note the robust post-inhibition rebound peak of activity (arrowhead). c) Average response of positivelyresponding neurons for various light pulse train frequencies delivered from the facing $\mu$ LED. Time basis is adapted to the period of stimulation. d) Same as c) for negatively-responding neurons. Note the shift on the $\mathrm{x}$-axis (phase) of the post-inhibitory rebound (arrowheads) for frequencies below $16 \mathrm{~Hz}$. e) Average neuronal firing modulation ( \pm SEM) at various distances from the facing $\mu \mathrm{LED}$ (negative values on the $\mathrm{x}$-axis indicate responses of neuron below the $\mu$ LED) for negatively and positively responding population of neurons for two example stimulation frequencies: 0.5 and $60 \mathrm{~Hz}$. Horizontal color-coded bars indicate values significantly different from 0 ( $\mathrm{p}<0.05$, Wilcoxon rank sum test). f) Average firing modulation ( \pm SEM) of positively (red) and negatively (blue) responding neurons to different light stimulation frequencies (x-axis). Horizontal bars indicate frequencies at which the modulation was significantly different from $0(\mathrm{p}<0.05$, Wilcoxon rank sum test).

\section{PV neurons inhibit cortical neurons in a layer-dependent manner}

Because connectivity patterns, neuronal density, membrane properties and post-synaptic targets of local PV neurons may differ from one layer to another (Buchanan et al., 2012; Bortone et al., 2014; Xue et al., 2014; Hafner et al., 2019; Vecchia et al., 2020), their activation might have a different effect according to their location in the cortical column. We therefore investigated whether there was a difference in rhythmic entrainment of S1 neocortical neurons by PV cells located in supragranular layers 2-3 vs. infragranular layers 5-6 when light was delivered locally vs in the opposite layers. Recorded neurons located above $-400 \mu \mathrm{m}$, or below $-500 \mu \mathrm{m}$ depths (Figure 1f) were considered as belonging to supragranular, or infragranular layers respectively (Lefort et al., 2009). The light was shone with equal intensity from each $\mu$ LED across layers and the modulation index was not significantly different between positively responding neurons from the 2 compartments, suggesting that the optical stimulation was delivering the same amount of excitation to PV positive neurons at all depths. 
As expected, negatively-responding RS neurons of both supra- and infra-granular layers (supra$\mathrm{RS}$ and infra-RS respectively) fired preferentially during $\mu \mathrm{LED}$ 'OFF' times (Figure 3a, for example at $4 \mathrm{~Hz}$ the mean firing phase of infra-RS was $4.61 \pm 0.05 \mathrm{rad}(\mathrm{p}<0.001, \mathrm{n}=88$, Rayleigh test). Deep layer neurons fired significantly earlier after the stimulation offsets than those of upper layers for most slow-wave and $\delta$ frequency stimulations $(0.5,1$ and 3 to $5 \mathrm{~Hz}$; Figure 3a; for example at 4Hz, infra-RS: $4.6 \pm 0.05 \mathrm{rad}, \mathrm{n}=88$; supra-RS: $4.8 \pm 0.05 \mathrm{rad}, \mathrm{n}=63 ; \mathrm{p}<0.001$, Kuiper test). WhenErreur! Signet non défini. the stimulation frequency increased, the preferred phase of firing was progressively delayed until the rebound-mediated activity couldn't follow the imposed rhythm above $18 \mathrm{~Hz}$, marking the maximum firing phase (arrowhead in Figure 3a). Negatively-responding supra-RS and infra-RS firing were significantly modulated for most stimulation frequencies when the light was delivered within their respective layer (Figure $3 b$ ). Interestingly their modulation index had a bimodal distribution relative to frequency. The two local maxima at $4-5 \mathrm{~Hz}$ and $\sim 35 \mathrm{~Hz}$ showed significant modulation of supra-RS neurons while the distribution was significantly bimodal (Hartigan dip test, $\mathrm{p}<0.001$ ), whereas light stimulations at $20 \mathrm{~Hz}$ showed a minimum and failed to evoke significant firing rate modulation (arrow in Figure 3b, infra-RS: $0.18 \pm 0.08, p>0.05, n=71$; supra-RS: $0.23 \pm 0.09, p>0.05, n=37$ ). These peaks and drops in rhythmic inhibition may be the resultant effect of network resonance effects. Indeed, at $\delta$ - $\theta$ frequencies, a sag in their firing rates could be observed at light stimulus onsets (Figure $3 b$ inset, double-arrow), indicating the activation of a rectifying current such as $I_{h}$ in response to hyperpolarizing inhibition. Therefore the higher efficiency for slow-wave and $\delta$-frequency stimulations to modulate supra-RS compared to infra-RS firing (figure $3 b$ and $3 b$ inset), could be explained by the differently pronounced sag-like shape of their neuronal responses (Figure $3 b$ inset). Infragranular layer pyramidal have been shown to exhibit stronger $\mathrm{I}_{\mathrm{h}}$-mediated currents than their supragranular counterparts (Berger et al., 2001; Larkum et al., 2007). Above $20 \mathrm{~Hz}$, the firing was significantly phased and modulated, with some significant differences between supra-RS and infra-RS neurons (Figure 3a-b). We hypothesized that slightly heterogeneous mean phases of action potential firing within a given neuronal population could result in a non-significant firing rates modulation index (at $>18 \mathrm{~Hz}$ ) while maintaining individual neuronal coherence could be preserved. Individual neuronal coherences were found to be significantly different from 0 across all stimulation frequencies (Figure 3c), albeit reduced in the $\gamma$ frequency range. While supra-RS fired more coherently at low frequencies $(<5 \mathrm{~Hz})$, no significant difference was found between 
supra-RS and infra-RS for $\gamma$ frequency stimulations. This indicates that the modulation index differences observed between supra-RS and infra-RS at $\gamma$ frequencies (Figure 3b) were more likely due to heterogeneities in phase shifts among the neuron population than to a decrease in their rhythmic entrainment. Overall IErreur! Signet non défini.h current being heterogeneous across layers in mice (Berger et al., 2001; Larkum et al., 2007; Kalmbach et al., 2018) and exerting a powerful control of the phase of action potential firing during $\theta$ oscillations (Kwag and Paulsen, 2009; Borel et al., 2013), RS neuronal resonant properties could be responsible for phase and modulation strength differences observed across layers.

Negatively-responding FS-neurons (whether from infra- or supragranular layers (infra-FS and supra-FS respectively)), showed similar overall entrainment properties to RS neurons (Figure 3df). However, while their firing was also phase-modulated with respect to the stimulation frequency (for example, supra-FS at $50 \mathrm{~Hz}$; phase $=4.71 \pm 0.19 \mathrm{rad}, \mathrm{p}=0.0003, \mathrm{n}=40$ Rayleigh test) with a general preference for the 'OFF' period of the $\mu$ LED pulse train (Figure 3d), stimulations in the $\gamma$ range drove a rhythmic activity in infra-FS that led to spikes occurring during the 'ON' period and considerably shifted their phase preference (Figure $3 \mathrm{~d}$ and $3 \mathrm{e}$ inset ; at $45 \mathrm{~Hz} \mathrm{p}=0.01, \mathrm{n}=21$ and 31 neurons) Kuiper test) compared to lower stimulation frequencies. Infra-FS and supra-FS firing rates were comparably modulated by light pulses delivered at $\delta$ and $\theta$ frequencies (Figure 3e; infra-FS at $4 \mathrm{~Hz}$, modulation index $=-0.59 \pm 0.09, \mathrm{p}<0.0001, \mathrm{n}=18$, Wilcoxon rank sum test), and their modulation index similarly dropped at the cut-off frequency of $18 \mathrm{~Hz}$ (Figure $3 \mathrm{e}$ arrowhead). However, supra-FS regained significant spike rate modulation in the $\gamma$ frequency range (Figure 3e, supra-FS at $50 \mathrm{~Hz}$ modulation index $=-0.34 \pm 0.1, \mathrm{p}<0.0001, \mathrm{n}=33$, infra-FS at $50 \mathrm{~Hz}$ modulation index $=0.08 \pm 0.18, \mathrm{p}=0.39, \mathrm{n}=21$, Wilcoxon rank sum test). Coherence analysis indicated that this layer-specific entrainment by light at $\gamma$ frequencies was mostly driven by phase difference heterogeneity, since coherence remained significantly high for both neuronal populations across all stimulation frequencies (Figure 3f). The lack of difference in modulation index at slow-wave and $\delta$ frequencies between supra-FS and infra-FS could be linked to the lack of low-frequency resonance in cortical FS neurons (Hutcheon et al., 1996). 

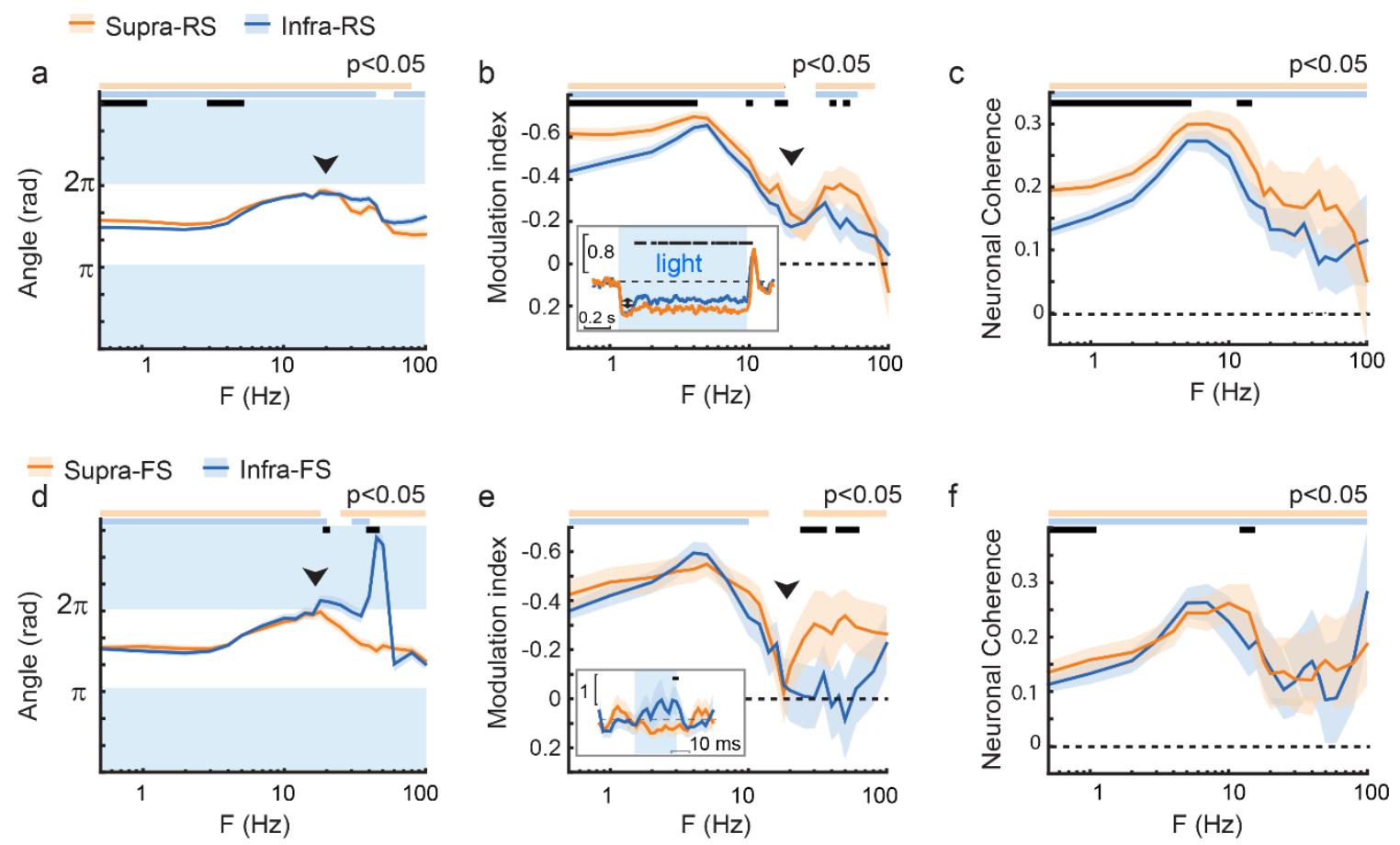

Figure 3: Frequency-dependent entrainment of neocortical neurons by optogenetic stimulation of PV neurons. a) Firing phase (mean \pm SEM) of inhibited RS neurons from the infragranular (blue) and supra-granular (orange) layers (inverted y-axis). Blue shaded area indicate the presence of $\mu L E D$ stimulation. Horizontal color bars indicate significant phasing (p-value $<0.05$, Rayleigh test). Black horizontal bars indicate significant phase difference between infra and supraRS ( $p<0.05$, Kuiper test). b) Average modulation index (mean \pm SEM) of RS neurons (same color-code as in a). Horizontal color bars indicate p-values $<0.05$ (Wilcoxon one-sided signedrank test). Black bars indicate significant difference between infra-RS (between 63 and 86 neurons) and supra-RS (between 38 and 57 neurons). Inset indicates the grand average normalized response (mean \pm SEM $n=73$ and 57 neurons for the $0.5 \mathrm{~Hz}$ stimulation). Note the significant difference (horizontal black bar, $\mathrm{p}<0.05$, one-sided signed-rank test) between infra-RS ( $\mathrm{n}=73$ ) and supra-RS $(n=48)$ responses. $c)$ Firing coherence across neurons and stimulation frequencies. (p-value $<0.05$, Wilcoxon rank sum test) for supra-RS and infra-RS. d-f) Same as (a-c) for FS neurons. In e, inset shows normalized firing rates (mean \pm SEM) during the pulsed stimulation at $45 \mathrm{~Hz}$ for infra-FS $(\mathrm{n}=24)$ and supra-FS $(\mathrm{n}=31)$ neurons. In $\mathrm{b}$ and e, significant bimodal distributions were found for supragranular modulation indexes (Hartigan dip test, $\mathrm{p}<0.001$ ). 
Next, we investigated how neuronal firing was affected depending on the layer at which local light stimulation occurred. Supra-RS neurons were preferentially modulated by supragranular-light stimulation (Supra-stim) at all stimulations below $10 \mathrm{~Hz}$ frequencies (Figure 4a, at $4 \mathrm{~Hz}$, modulation $=-0.70$ vs $-0.47 \mathrm{p}<0.0001, \mathrm{n}=63$ and 51 neurons, Wilcoxon rank sum test $)$ and at some $\gamma$ frequencies i.e. $50 \mathrm{~Hz}$ and $60 \mathrm{~Hz}$. In contrast, infra-RS were modulated as strongly by light stimulations occurring within the same layer as from the opposite layer at most stimulation frequencies (Figure 4c, $n=88$ and 61 neurons, Wilcoxon rank sum test). This indicates that PVmediated inhibition spreads more efficiently from upper to deeper layers than in the opposite direction. Optogenetic stimulation of PV neurons significantly modulated neuronal firing phases for most stimulation frequencies irrespective of the location of the light stimulation (Figure 4b, d). Overall, the inhibition generated through PV-neuron rhythmic activation efficiently spread to RS neurons in other layers essentially when the stimulation frequency was below $\sim \leq 10 \mathrm{~Hz}$ and better in the descending way.

While supra-FS were similar to supra-RS as their modulation was significantly stronger when light was shone on the same layer compared to opposite layer (modulation=-0.53 vs $-0.34, p=0.0025$, $n=31$ and 36 neurons, Wilcoxon signed-rank test) (Figure 4e), infra-FS contrasted with infra-RS because they showed a stronger modulation for same-layer stimulations (modulation $=0.59$ vs 0.36 , $\mathrm{p}=0.022, \mathrm{n}=18$ and 11 neurons, Figure $4 \mathrm{~g}$ ). This indicates that infragranular PV-mediated inhibition tends to remain more layer-specific for FS-neurons than it is for RS-neurons (Figure $4 \mathrm{~g}$ vs. 4c) for infragranular neurons. Lastly, the phase of firing could be differently modulated when light stimulations were delivered from the opposite layers but most absolute phase differences remained small (Figure 4f, h). 


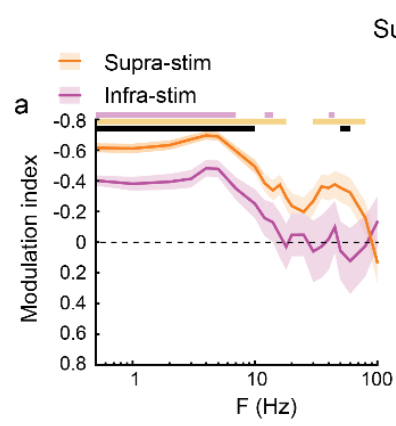

Supra-RS
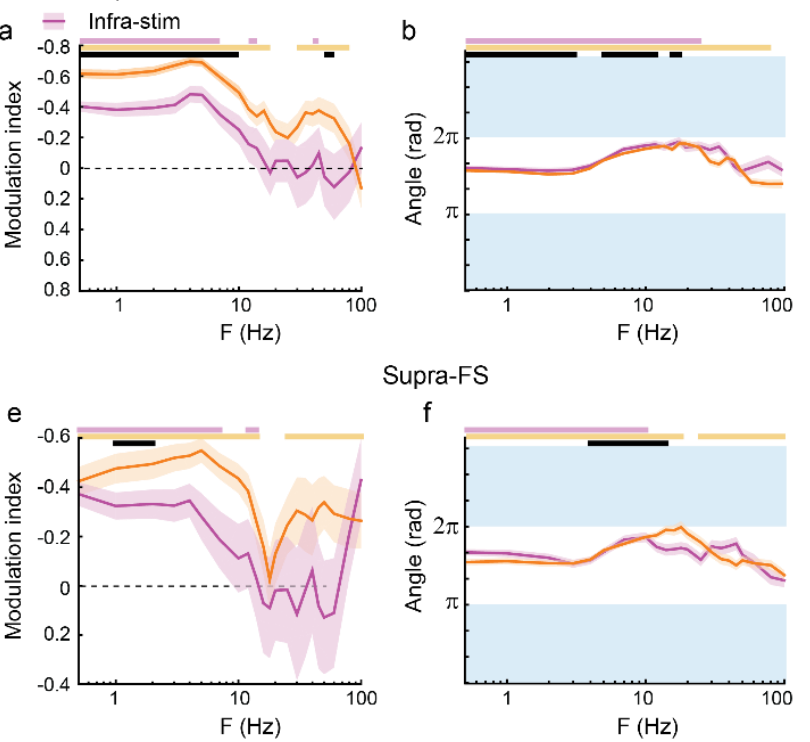

$f$

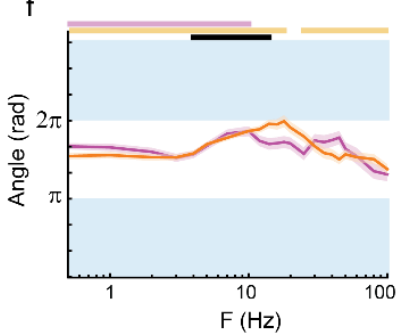

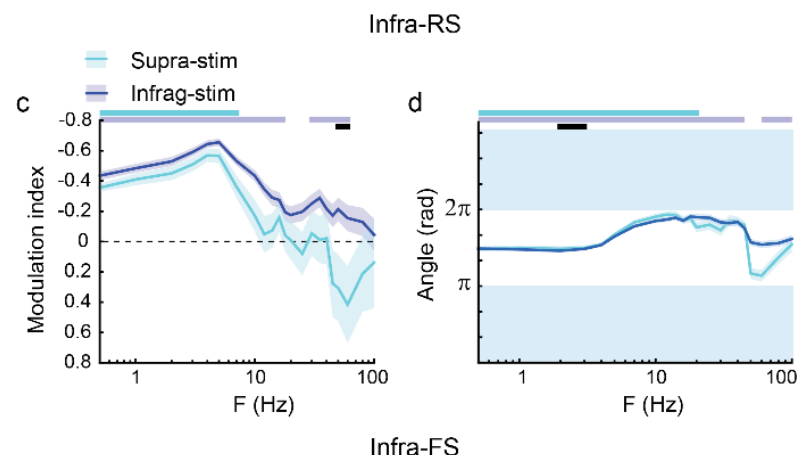

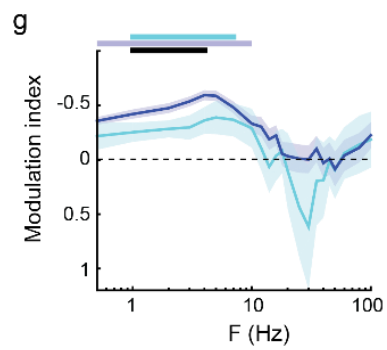

$\mathrm{h}$

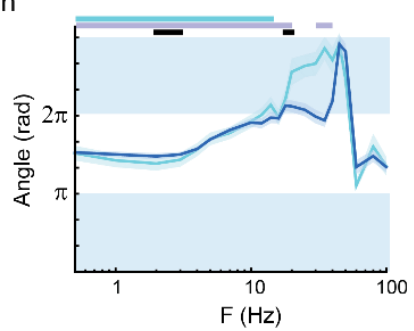

Figure 4. Translaminar effect of PV optogenetic activation on RS and FS firing. a) Modulation index (mean \pm SEM) for supra-RS neurons in response to cross-layer (purple) or samelayer stimulation (orange). Note the significant difference of the modulation index between supra and infragranular layers stimulation at $\leq 10 \mathrm{~Hz}$. (Horizontal black bar, $\mathrm{p}<0.05$, Wilcoxon rank sum test). b) Firing phase (mean \pm SEM) of supra-RS neurons for cross-layer and same-layer stimulations. c, d) Same as (a,b) for infra-RS neurons. Note the lack of significant difference of the modulation index between supra and infragranular layers stimulation at $\leq 10 \mathrm{~Hz}$. e-h) Same as (a-d) for Supra-FS and infra-FS. Only consecutive $\mathrm{p}<0.05$ on the $\mathrm{x}$-axis are presented for simplicity.

\section{LFP rhythms induced by PV-neuron activation reveal complex network interactions}

Cortical LFP oscillatory activity is often associated with specific cognitive processes or vigilance states (Destexhe et al., 1999; Urbain et al., 2019), and we therefore next investigated how rhythmic cortical PV neuronal activation impacted their activity. When stimulations were applied at $\delta-\theta$ frequencies, both infra- and supra- LFP signals displayed an increase in oscillation amplitude at the frequency of stimulation, independently of the location at which light was applied (Figure 5a). However, the increase was reduced at $\gamma$ frequencies, (Figure 5b). In order to compare the impact of these stimulations on supra- and infra-LFPs, we calculated the LFP modulation index, based on 
a differential measure of the LFPs which increases the net effects induced by local stimulation while decreasing distant rhythm generator effects (Kajikawa and Schroeder, 2011, see Methods). This LFP modulation index increased significantly at $\delta$ frequencies, irrespective of both the depth at which the light was applied and the LFP recording site e.g. supragranular (Figure 5c left, maximal modulation index $=3.57 \pm 0.53$ at $-700 \mu \mathrm{m}, \mathrm{n}=24$ trials, $\mathrm{p}<0.0001$, Wilcoxon rank sum test) or infragranular (Figure 5c right, maximum modulation index of $4.12 \pm 0.18$ at $-900 \mu \mathrm{m}, \mathrm{n}=$ 28 trials, $\mathrm{p}<0.0001$, Wilcoxon rank sum test). The average infra-LFP modulation index at $\delta$ frequencies was however significantly higher when the light stimulation was applied at the level of infragranular layers (Figure 5c right, modulation indexes $1.81 \pm 0.12$ at $-100 \mu \mathrm{m}$ vs. $4.12 \pm 0.18$ at $-900 \mu \mathrm{m}, \mathrm{p}<0.0001$, Wilcoxon rank sum test, $\left.\mathrm{n}_{1}=28, \mathrm{n}_{2}=24\right)$, indicating a preferential depth for $\delta$ frequency oscillations amplification through activation of local PV neurons. Overall, both supra- and infra-LFP modulation indexes were significantly linearly correlated with the stimulation depth (Figure 5c, linear regression: slope $=-0.31, \mathrm{r}^{2}=0.20, \mathrm{p}<0.0001$ for infra-LFPs and slope $=-0.02, \mathrm{r}^{2}=0.02, \mathrm{p}<0.05$ for supra LFPs). The same stimulation-depth-dependent LFP modulation was observed and significant but not represented here at $\theta$ frequency stimulations.

On the other hand, when considering the impact of stimulations performed at $\gamma$-frequencies, we found that local activation of PV neurons resulted in larger infra- and supra-LFP modulation indexes for almost all stimulation depths (Figure 5d). However, only Supra-LFP modulation was linearly correlated with the stimulation depth (Figure 5d left, supra-LFP modulation index at -300 $\mu \mathrm{m}=0.85 \pm 0.15, \mathrm{p}<0.0001, \mathrm{n}=30$ trials, significantly higher than at infragranular depths of stimulation; supra-LFP modulation index at $-700 \mu \mathrm{m}=0.38 \pm 0.16, \mathrm{p}=0.031, \mathrm{n}=41$ trials; linear regression: slope $\left.=0.12, \mathrm{r}^{2}=0.07, \mathrm{p}<0.0001\right)$.

At $\delta-\theta$ frequencies, the broader impact of local light stimulation on LFP modulation indexes show a peak emerging at $5 \mathrm{~Hz}$ (Figure $5 \mathrm{e}-\mathrm{g}$ ), similar to the one we reported for firing modulation indexes of RS and FS neurons (Figure 3b, e). Surprisingly however, the cortical depth for light stimulation at which we observed maximal LFP modulation indexes was different from the supra-granular position observed for the maximal RS-neuron modulation (see Figure 3b). The LFP modulation index in the $\gamma$ frequency range at 35, 40 and $80 \mathrm{~Hz}$ was higher for supra- versus infra-LFP, when the stimulation was delivered in the respective layers (Figure 5e). Translaminar vs local modulation displayed a significantly stronger supra-LFP modulation when the stimulation was 
delivered within the supragranular vs. the infragranular layer at $35 \mathrm{~Hz}$ and $40 \mathrm{~Hz}$ (Figure 5f, supraLFP modulation index at $35 \mathrm{~Hz}$, supra-stimulation $1.02 \pm 0.14$ vs infragranular stimulation $0.22 \pm$ 0.09, $\mathrm{p}=0.013$, ( $\mathrm{n}=9$ and 19) Wilcoxon rank sum test). In contrast, supragranular layer stimulation did not have any stronger impact than infragranular stimulation when the LFP was recorded in the infragranular layer (Figure $5 \mathrm{~g}$ ). Taken together, our results indicate that $\delta-\theta$ oscillations are particularly well entrained throughout a cortical column when the stimulation is in infragranular layers, while $\gamma$ rhythms are best entrained by local stimulation of supragranular PV neurons with less layer-specific effect.

a

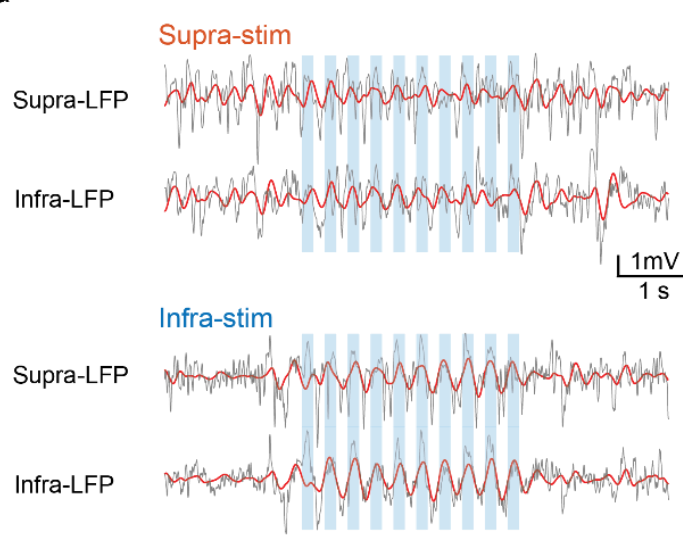

b
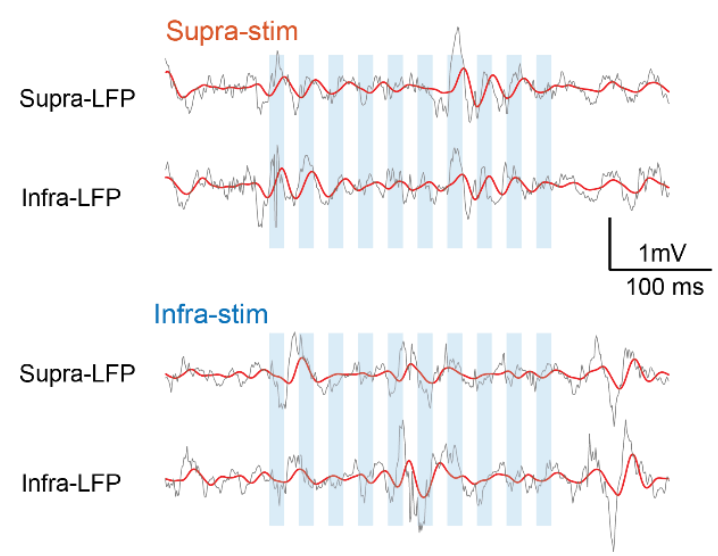

C DELTA Supra-LFP

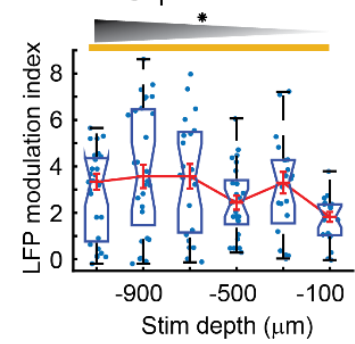

e

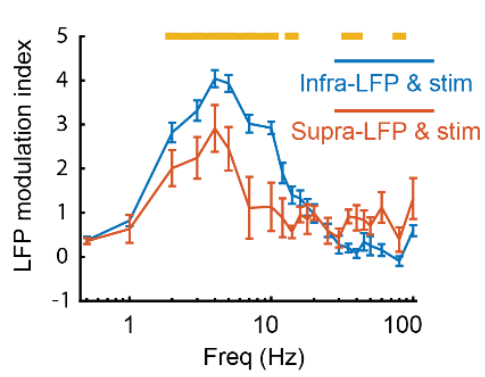

Infra-LFP

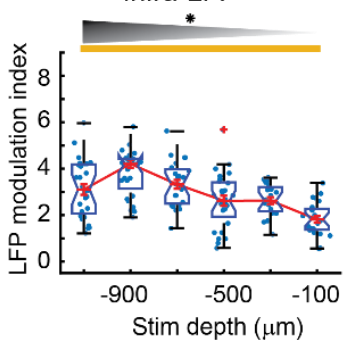

d GAMMA Supra-LFP

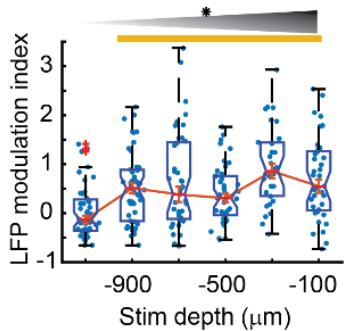

Infra-LFP

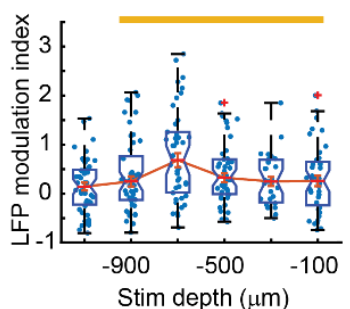

f

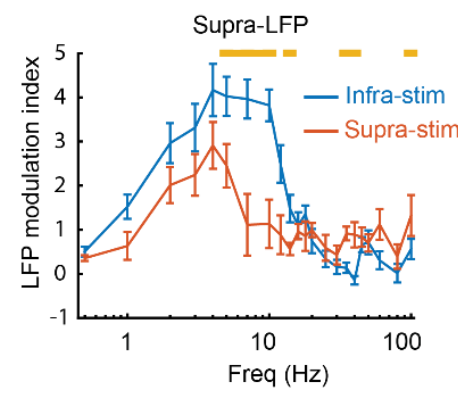

g

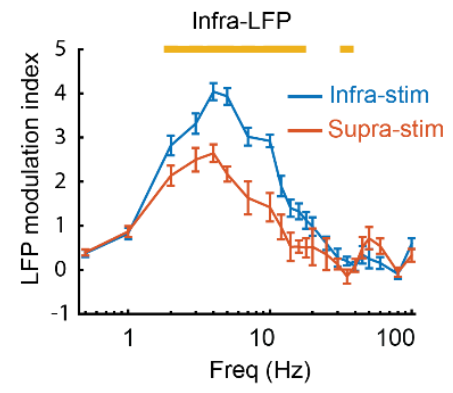

Figure 5: Laminar control of cortical LFP oscillations by activation of PV neurons a) Example supra- and infra-granular LFP traces during $3 \mathrm{~Hz}$ optogenetic activation of supra- and 
infragranular PV neurons. Infragranular stimulations induced higher amplitude oscillations compared to supragranular layer stimulations. b) Same as a) for $25 \mathrm{~Hz}$ activation of PV neurons. c) Median supra- (left) and infra- (right) LFP modulation index in response to $\delta(2-5 \mathrm{~Hz})$ frequency stimulation. Boxes indicate the $1^{\text {st }}$ and $3^{\text {rd }}$ quartiles, notches extrema and red crosses outliers. Black gradient and stars indicate significant linear correlation between LFP modulation index and stimulation depth (supra-LFP: $\mathrm{p}=0.0074, \mathrm{n}=124$; infra-LFP $\mathrm{p}<0.0001, \mathrm{n}=128$ (infragranular)). Yellow bars indicate significant modulation at each depth $(\mathrm{p}<0.05$, Wilcoxon rank sum test). d) Same as c) for $\gamma(25-80 \mathrm{~Hz})$ frequency stimulations. e) Modulation index curve of infra-LFP (blue), supra-LFP (red) in response to infra-and supragranular light stimulations. f) Comparison of supraLFP modulation indexes for infra- (blue) and supra- (red) granular light stimulations. Yellow bars indicate significant differences ( $\mathrm{p}<0.05$, Wilcoxon signed-rank test)) $\mathrm{g}$ ) Comparison of infra-LFP modulation indexes for infra-(blue) and supra-(red) granular light stimulations.

\section{Discussion}

We investigated the impact of layer-rhythmic optogenetic activation of PV neurons on neuronal and network activity within a column of the mouse barrel somatosensory neocortex in nonanesthetized awake animals. Specifically, we asked whether supra- and infra-granular PV populations were more or less prone to entrain certain rhythmic oscillations within a cortical column and how such entrainments might spread across its layers. We found common frequency preferences in the range of $2-16 \mathrm{~Hz}$ for oscillatory entrainment observed at the level of the LFP, as well as neuronal firing of FS and RS neurons, in accordance with a previous report using chirplike light pulses in freely-moving animals (Stark et al., 2013). However, we observed a peak resonance at $4-5 \mathrm{~Hz}$ in the $\delta$ range rather than the $\theta$ range, presumably because our animals were head-fixed and not engaged in active spatial exploration (Stark et al., 2013). Furthermore, in our study, our use of layer-restricted square light pulses resulted in the activation of a spatiallyrestricted pool of PV neurons, confirmed by the observation that at $\delta-\theta$ frequencies, neuronal firing rates modulations were indeed spatially localized, i.e. stronger at the level of the layer where light was applied than elsewhere. Despite this spatially-restricted activation of local PV neurons, we found that the induced low-frequency inhibition could spread beyond the activated layer in both 
directions (ie: infragranular-to-supragranular layers and vice-versa). In comparison, $\gamma$-frequency stimulations entrained LFP and neuronal overall firing rates with much less amplitude.

We also found a layer-specific and frequency-dependent entrainment of LFP oscillations and neuronal firing rates modulations. At $\delta$ frequencies, a higher LFP modulation was observed when infragranular PV neurons were rhythmically activated through optogenetic light stimulations, whereas at $\gamma$ frequencies activation of supragranular PV neurons evoked the highest modulation. Similarly, supra-RS neurons firing rates were more strongly modulated than infra-RS at $\delta$ frequencies (Figure 3b,c) while infra-RS neurons were more selective than their supra-RS counterparts to the peak frequency of entrainment. Finally, at $\gamma$ frequencies, the firing rates of supra-FS neurons were more strongly modulated compared to their infra-FS, counterparts. The layer-specific LFP entrainment properties in non-anesthetized mice that we uncovered match some previously reported layer-specific properties of cortical rhythm generation, notably that deep layer neurons are capable of generating $\delta$ waves (Carracedo et al., 2013) while upper cortical layers can synchronize at $\gamma$ frequencies (Buffalo et al., 2011). However, in our hands, no direct link could be established between LFP and neuronal firing modulations since LFPs were overall better entrained within deep layers whereas supra-RS firing was better amplified than infra-RS. We propose hereby some interpretation of the results, based on two different mechanisms - neuronal entrainment and firing resonance (Richardson et al., 2003; Wilson et al., 2018; Lakatos et al., 2019).

\section{Frequency-band and layer-specific amplification of cortical rhythms}

We showed that PV-driven LFP rhythms could reliably spread across layers at physiological frequencies $(0.5-100 \mathrm{~Hz})$, but that there was a directional preference for infra- to supragranular layers in the $\delta / \theta$ frequency range and for supra- to infragranular layers in the $\gamma$ frequency range (Figure 5). Previous in vitro studies investigating supra- and infra-granular layer contributions to low frequency activity revealed the prominent role for infragranular cortex in maintaining low frequency cortical oscillations (Sanchez-Vives and McCormick, 2000; Hirata and CastroAlamancos, 2010; Wester and Contreras, 2012). Similarly, slow oscillations in the barrel cortex of the mouse were preferentially generated in layer 5 in anesthetized animals (Beltramo et al., 2013). Our results extend this finding by showing that these slow rhythms, once present in layer 5, spread more efficiently to supragranular layers. Infragranular layers, which include the main cortical 
output layer 5, therefore appear to be involved in both the generation and transfer of low-frequency oscillations within a cortical column. In contrast, the neuron firing analysis indicated that the rhythmic neuronal inhibition spreads better from upper to deeper layers in the $\delta / \theta$ frequency range than the way around (Figure $4 \mathrm{~b}-\mathrm{f}$ ), possibly due to the more abundant functional connectivity from supragranular layers to infragranular layers (Senzai et al., 2019).

Compared to previous studies (Cardin et al., 2009 ; Kuki et al., 2012), our use of $\mu$ LED arrays allowed us to disentangle infra- from supra-granular networks and to investigate the spread of cortical rhythms throughout a cortical column. In addition to the broader range of entrainment frequencies we applied, we reported a stronger role of the supragranular layer circuit and PVmediated rhythms at $\delta / \theta$ frequencies compared to previous reports. In their study, Beltramo et al (2013), reported an almost exclusive impact of the infragranular excitatory circuit at low frequencies, and Cardin et al. (2009), did not report any impact on the LFP from PV neurons at low frequencies. The differences between their results and ours could be due to the anesthetized vs awake conditions. Indeed, anaesthesia via norepinephrine reduction has been shown to shunt apical dendritic communication with the soma through $I_{h}$ activation (Phillips et al., 2016). Furthermore, some anaesthetics also block directly amplification currents such as those emanating from T-type calcium channels activation (Timic Stamenic et al., 2019) and $I_{h}$ inward cation nonspecific current (Zhou et al., 2012). Instead, under awake conditions, inhibitory inputs within the supragranular layer could be more efficient at gating not only Layer 2/3 neurons but also L5 neurons through their apical dendritic arborization into layers 1-3. Overall, this would result in the widespread inhibitory modulation observed in our conditions.

At $\gamma$ frequencies, the widespread entrainment across layers that we observed could be explained by the strong connectivity within the cortical column and the presence of current sources and sinks of $\gamma$ oscillations all along cortical columns of the neocortex (Senzai et al., 2019). Interestingly, the differences in $\gamma$ oscillation amplification compared to $\delta-\theta$ could also be due to the lack of postinhibitory peaks of activity after each pulse of inhibition above $18 \mathrm{~Hz}$ (Figure 2b,d), thereby blocking a major boost to low frequency rhythms via resonant and amplification mechanisms. This decrease in amplitude, equivalent to a high-frequency cut-off, is often masked by the 1 /frequency decrease of EEG/LFP power (Vinck et al., 2015). It could nevertheless reflects physiological mechanisms that also exist within the spontaneous LFP activity (see for instance 'debiased' LFP 
representations in Figure 1 of Vinck et al., 2015) and limit local network synchronization above $18 \mathrm{~Hz}$.

Neuronal circuit correlates of layer-specific $\delta$ - $\theta$ amplification

The average neuronal firing rate correlates well with the LFP in anesthetized (Volgushev et al., 2006; Beltramo et al., 2013) and behaving animals (Csicsvari et al., 2003) as the recurrent neuronal communication within the cortical column is assumed to be a major contributor to cell assemblies (Cohen-Kashi Malina et al., 2016). Firing rates can also be used as a proxy of membrane potential dynamics (Shriki et al., 2003; Schaffer et al., 2013). In our study, the impact of pulsed inhibition onto the neuronal firing unravelled the presence of different firing rate peaks that were dependent on the neuronal cell type, the pulsed frequency and the cortical layer. Various cell types have been shown to display different intrinsic resonant properties (Ledoux and Brunel, 2011; Bel and Rotstein, 2019). At the neuronal level, the combination of post-inhibitory rebound (Llinás and Jahnsen, 1982) and sag currents (McCormick and Pape, 1990), is a source of membrane potential resonance in the thalamus (Leresche et al., 1991; Soltesz et al., 1991; Lüthi and McCormick, 1998; Ulrich, 2014) and the cortex (Ulrich, 2002; Kalmbach et al., 2018). Here, those electrophysiological signatures were found on the pulse-triggered averages, which showed both a post-inhibitory rebound in firing (Figure 2b, d) and the hyperpolarization-induced increased in activity (Figure 3b inset). Both T-type calcium channels and hyperpolarization activated cyclic nucleotide (HCN) channels are present in pyramidal neurons of the neocortex (Talley et al., 1999; Berger et al., 2001). We found a stronger component of this HCN-like mediated activity in the infra-RS subpopulation (Figure $3 \mathrm{~b}$ inset), which coincides with the location where the stimulation led to stronger LFP amplification. This observation also mirrors the differential distribution of $\mathrm{I}_{\mathrm{h}}$ channels observed in neocortex (Berger et al., 2001; Larkum et al., 2007; Kalmbach et al., 2018), indicating a potential contributing role of $I_{h}$ in the entrainment of neocortical $\delta$ and $\theta$ rhythms (Stark et al., 2013). Notwithstanding those neuronal correlates of LFP layer-specific amplification, the layer-specific effect of PV neuron activation could also reflect some layer-specific perisomatic connections with pyramidal neurons and axonal translaminar projections (Buchanan et al., 2012; Bortone et al., 2014; Vecchia et al., 2020). Moreover, PV neurons synapses onto basal dendrites of RS neurons can locally impair local excitatory input summation in particular at low frequencies (Branco and Häusser, 2011) and interact with HCN-channel-rich dendrites (Lörincz et al., 2002). 
How then can the greater observed impact of stimulations on both supra RS neurons but infra-LFP be reconciled?

Multiples mechanisms could be involved. 1) A minority of neurons not visible at the population level with an uneven distribution between intra- and supra-granular layers would result in a stimulation position-dependent effect. Many neuron types could fit in the requirement of such layer-specific effect like Martinotti cells, somatostatin neurons (Muñoz et al., 2017), or the vasointestinal peptide positive neurons as they have an asymmetric distribution across cortical layers (Tremblay et al., 2016) and represent relatively few neurons among the cortical neuronal population. 2) Some specific synapses properties present in infragranular or supragranular layers could also bias the effect of PV-neuron mediated inhibition toward some specific layers. Such possibility remains relatively unexplored due to scarcity of the tools to probe synapses (Adesnik and Naka, 2018). Last but not least, 3) neuronal intrinsic resonant properties (Kalmbach et al., 2018) could underlie circuit mechanisms not accessible with our tools and explain the differential impact between neuronal and LFP entrainment.

\section{Neuronal and network $\delta$ - $\theta$ oscillation layer-specific mechanisms}

Neuronal modulations were slightly stronger at $\delta$ frequencies in supragranular compared to infragranular layers (Figure 3b-c). However, the LFP modulation was much stronger when the stimulation was applied in infragranular layers (Figure 5). Despite observed and known intrinsic neuronal differences across layers, one element differed noticeably for neuronal entrainment between layers i.e. the selectivity of the neurons to the frequency of stimulation. Yet this firing frequency selectivity, associated with intrinsic membrane potential resonance properties (Wang, 2010), is a good indicator of how the neurons can influence network oscillations (Bel and Rotstein, 2019). In the $\delta$ frequency range both RS and FS neurons have a specific 'bell shape" selectivity of responses across stimulation frequencies that is sharper in infragranular than in supragranular layers (Figure 3b,c,e,f). This frequency selectivity is a key element of neuronal entrainment as it tends to narrow down the range at which the network can effectively be entrained (Bel and Rotstein, 2019). HCN channels have been shown to underlie membrane potential frequency resonance (Hutcheon et al., 1996) and $\theta$ frequency entrainment in vivo (Stark et al., 2013). Contrary to RS neurons, the origin of similar properties in FS neurons (Figure 3e,f) is not clear, as 
it does not reflect their intrinsic property (since FS neurons do not have resonance in the $\delta-\theta$ band (Hutcheon et al., 1996)). It more likely matches the network resonance as FS neurons quickly counterbalance RS neuronal activity. In addition to neuron-resonance based mechanisms, the dense lateral excitatory connectivity at the level of layer 5 (Dantzker and Callaway, 2000; Schubert et al., 2007; Wester and Contreras, 2012) between pyramidal neurons and the corticothalamic connectivity may contribute to a higher rebound network-mediated amplification of the $\delta$ - $\theta$ cortical rhythms, while not being visible at the local neocortical population level. In contrast, in supragranular layers, the less efficient filtering of the frequency response, the sparser lateral connectivity of layer 2-3 neurons could induce a relative failure to amplify the $\delta-\theta$ rhythms at the network level.

Among other factors than resonance, the peak frequency observed in vitro (Hutcheon et al., 1996) or in vivo (Stark et al., 2013), could be under the control of physiological parameters such as connectivity, temperature and neuromodulatory tone. Those parameters may explain also how the same PV-mediated layer-specific mechanisms can contribute to the emergence of multiple layerdependent neocortical rhythms as diverse as the hippocampal-related neocortical $\theta$ rhythms (Sirota et al., 2008), the rapid-eye-movement (REM) sleep $\theta$ rhythm, the $\alpha$-rhythm, the sleep spindles, non-REM sleep thalamic-related $\delta$ rhythms or some forms of absence epilepsy (Crunelli et al., 2020) which all involve neocortical PV-neuron rhythmic activity in this frequency band.

\section{Neuronal and network $\gamma$ oscillation dynamic layer specific mechanisms}

In this study, we observed a significantly higher modulation of firing in FS neurons in supragranular layer at $\gamma$ frequencies. In our preparation, not all PV neurons expressed ChR2, and PV-positive responding neurons (mostly FS neurons) could inhibit FS neurons via FS-to-FS interconnections (Tamás et al., 1998; Galarreta and Hestrin, 2002), and influence the network dynamics at the $\gamma$ frequency as previously proposed as one of the possible mechanisms for $\gamma$ oscillation synchronization (Whittington et al., 2000; Börgers and Kopell, 2003). Indeed, our observed peak for supragranular FS neuronal modulation at $\gamma$ frequencies matches the frequency band of maximal coherence between spikes and LFP (Perrenoud et al., 2016) and the frequency band at which PV neurons stimulation triggers maximal increase in LFP power spectra (Cardin et al., 2009). This cortical network property could result from the subthreshold resonant and spiking 
resonant properties which are both present in cortical PV neurons (Tikidji-Hamburyan et al., 2015). The peak of FS neuron modulation at $\gamma$ frequencies was observed only in the supragranular layer. Although this layer-specific property was surprising, infragranular FS-neuron intrinsic functional properties are still considerably less well characterized. The existence of two populations of cortical infra- and supragranular layer PV neurons that are differentially equipped to locally sustain PV-driven $\gamma$ rhythms would allow more specialized computations to take place within a functional column, while maintaining the interactions necessary to sustain interlaminar information transfer.

Acknowledgements: The project was funded by the European Union 7th Framework Program (FP7/2007-2013) under grant agreement n॰600925 (NeuroSeeker).

\section{References:}

Adesnik H, Naka A (2018) Cracking the Function of Layers in the Sensory Cortex. Neuron 100:1028-1043.

Ahissar E, Staiger J (2010) S1 laminar specialization. Scholarpedia 5:7457.

Arnal LH, Giraud A-L (2012) Cortical oscillations and sensory predictions. Trends Cogn Sci (Regul Ed) 16:390-398.

Ayub S, David F, Klein E, Borel M, Paul O, Gentet L, Ruther P (2020) Compact optical neural probes with up to 20 integrated thin-film \$Imu\$LEDs applied in acute optogenetic studies. IEEE Transactions on Biomedical Engineering:1-1.

Ayub S, Gentet LJ, Fiáth R, Schwaerzle M, Borel M, David F, Barthó P, Ulbert I, Paul O, Ruther P (2017) Hybrid intracerebral probe with integrated bare LED chips for optogenetic studies. Biomedical Microdevices 19:49.

Barthó P, Hirase H, Monconduit L, Zugaro M, Harris KD, Buzsáki G (2004) Characterization of neocortical principal cells and interneurons by network interactions and extracellular features. Journal of neurophysiology 92:600-608.

Bastos AM, Vezoli J, Bosman CA, Schoffelen J-M, Oostenveld R, Dowdall JR, Weerd PD, Kennedy H, Fries P (2015) Visual Areas Exert Feedforward and Feedback Influences through Distinct Frequency Channels. Neuron 85:390-401. 
Bel A, Rotstein HG (2019) Membrane potential resonance in non-oscillatory neurons interacts with synaptic connectivity to produce network oscillations. J Comput Neurosci 46:169195.

Beltramo R, D’Urso G, Maschio MD, Farisello P, Bovetti S, Clovis Y, Lassi G, Tucci V, Tonelli DDP, Fellin T (2013) Layer-specific excitatory circuits differentially control recurrent network dynamics in the neocortex. Nature neuroscience 16:227-234.

Berger T, Larkum ME, Lüscher HR (2001) High I(h) channel density in the distal apical dendrite of layer $\mathrm{V}$ pyramidal cells increases bidirectional attenuation of EPSPs. J Neurophysiol 85:855-868.

Borel M, Guadagna S, Jang HJ, Kwag J, Paulsen O (2013) Frequency dependence of CA3 spike phase response arising from h-current properties. Front Cell Neurosci 7:263.

Börgers C, Kopell N (2003) Synchronization in networks of excitatory and inhibitory neurons with sparse, random connectivity. Neural computation 15:509-538.

Bortone DS, Olsen SR, Scanziani M (2014) Translaminar inhibitory cells recruited by layer 6 corticothalamic neurons suppress visual cortex. Neuron 82:474-485.

Branco T, Häusser M (2011) Synaptic integration gradients in single cortical pyramidal cell dendrites. Neuron 69:885-892.

Buchanan KA, Blackman AV, Moreau AW, Elgar D, Costa RP, Lalanne T, Tudor Jones AA, Oyrer J, Sjöström PJ (2012) Target-specific expression of presynaptic NMDA receptors in neocortical microcircuits. Neuron 75:451-466.

Buffalo EA, Fries P, Landman R, Buschman TJ, Desimone R (2011) Laminar differences in gamma and alpha coherence in the ventral stream. Proc Natl Acad Sci U S A 108:1126211267.

Buzsáki G, Draguhn A (2004) Neuronal Oscillations in Cortical Networks. Science 304:19261929.

Buzsáki G, Wang X-J (2012) Mechanisms of Gamma Oscillations. Annual Review of Neuroscience 35:203-225.

Cardin JA, Carlén M, Meletis K, Knoblich U, Zhang F, Deisseroth K, Tsai L-H, Moore CI (2009) Driving fast-spiking cells induces gamma rhythm and controls sensory responses. Nature 459:663-667.

Carracedo LM, Kjeldsen H, Cunnington L, Jenkins A, Schofield I, Cunningham MO, Davies CH, Traub RD, Whittington MA (2013) A neocortical delta rhythm facilitates reciprocal interlaminar interactions via nested theta rhythms. J Neurosci 33:10750-10761.

Chauvette S, Seigneur J, Timofeev I (2012) Sleep oscillations in the thalamocortical system induce long-term neuronal plasticity. Neuron 75:1105-1113. 
Chen G, Zhang Y, Li X, Zhao X, Ye Q, Lin Y, Tao HW, Rasch MJ, Zhang X (2017) Distinct Inhibitory Circuits Orchestrate Cortical beta and gamma Band Oscillations. Neuron 96:1403-1418.e6.

Cohen-Kashi Malina K, Mohar B, Rappaport AN, Lampl I (2016) Local and thalamic origins of correlated ongoing and sensory-evoked cortical activities. Nat Commun 7:12740.

Crunelli V, Lőrincz ML, McCafferty C, Lambert RC, Leresche N, Di Giovanni G, David F (2020) Clinical and experimental insight into pathophysiology, comorbidity and therapy of absence seizures. Brain.

Csicsvari J, Henze DA, Jamieson B, Harris KD, Sirota A, Barthó P, Wise KD, Buzsáki G (2003) Massively parallel recording of unit and local field potentials with silicon-based electrodes. J Neurophysiol 90:1314-1323.

Dantzker JL, Callaway EM (2000) Laminar sources of synaptic input to cortical inhibitory interneurons and pyramidal neurons. Nat Neurosci 3:701-707.

Destexhe A, Contreras D, Steriade M (1999) Spatiotemporal analysis of local field potentials and unit discharges in cat cerebral cortex during natural wake and sleep states. J Neurosci 19:4595-4608.

Douglas RJ, Martin KAC (2004) Neuronal circuits of the neocortex. Annu Rev Neurosci 27:419-451.

Durkin J, Suresh AK, Colbath J, Broussard C, Wu J, Zochowski M, Aton SJ (2017) Cortically coordinated NREM thalamocortical oscillations play an essential, instructive role in visual system plasticity. Proc Natl Acad Sci U S A 114:10485-10490.

Ferguson BR, Gao W-J (2018) PV Interneurons: Critical Regulators of E/I Balance for Prefrontal Cortex-Dependent Behavior and Psychiatric Disorders. Front Neural Circuits 12 Available at: https://www.ncbi.nlm.nih.gov/pmc/articles/PMC5964203/ [Accessed December 13, 2020].

Fries P (2015) Rhythms for Cognition: Communication through Coherence. Neuron 88:220-235.

Galarreta M, Hestrin S (2002) Electrical and chemical synapses among parvalbumin fast-spiking GABAergic interneurons in adult mouse neocortex. Proc Natl Acad Sci U S A 99:1243812443.

Gentet LJ, Kremer Y, Taniguchi H, Huang ZJ, Staiger JF, Petersen CCH (2012) Unique functional properties of somatostatin-expressing GABAergic neurons in mouse barrel cortex. Nature neuroscience 15:607-612.

Hafner G, Witte M, Guy J, Subhashini N, Fenno LE, Ramakrishnan C, Kim YS, Deisseroth K, Callaway EM, Oberhuber M, Conzelmann K-K, Staiger JF (2019) Mapping Brain-Wide Afferent Inputs of Parvalbumin-Expressing GABAergic Neurons in Barrel Cortex Reveals Local and Long-Range Circuit Motifs. Cell Rep 28:3450-3461.e8. 
Hartigan JA, Hartigan PM (1985) The Dip Test of Unimodality. Ann Statist 13:70-84.

Herreras O (2016) Local Field Potentials: Myths and Misunderstandings. Front Neural Circuits 10:101.

Hu H, Gan J, Jonas P (2014) Interneurons. Fast-spiking, parvalbumin ${ }^{+}$GABAergic interneurons: from cellular design to microcircuit function. Science 345:1255263.

Hutcheon B, Miura RM, Puil E (1996) Subthreshold membrane resonance in neocortical neurons. J Neurophysiol 76:683-697.

Kajikawa Y, Schroeder CE (2011) How Local Is the Local Field Potential? Neuron 72:847-858.

Kalmbach BE et al. (2018) h-Channels Contribute to Divergent Intrinsic Membrane Properties of Supragranular Pyramidal Neurons in Human versus Mouse Cerebral Cortex. Neuron 100:1194-1208.e5.

Kawaguchi Y, Kubota Y (1997) GABAergic cell subtypes and their synaptic connections in rat frontal cortex. Cerebral Cortex 7:476-486.

Klausberger T, Marton LF, O’Neill J, Huck JHJ, Dalezios Y, Fuentealba P, Suen WY, Papp E, Kaneko T, Watanabe M, Csicsvari J, Somogyi P (2005) Complementary roles of cholecystokinin- and parvalbumin-expressing GABAergic neurons in hippocampal network oscillations. J Neurosci 25:9782-9793.

Kuki T, Ohshiro T, Ito S, Ji Z-G, Fukazawa Y, Matsuzaka Y, Yawo H, Mushiake H (2012) Frequency-dependent entrainment of neocortical slow oscillation to repeated optogenetic stimulation in the anesthetized rat. Neuroscience research 75:35-45.

Kwag J, Paulsen O (2009) Bidirectional control of spike timing by GABA(A) receptor-mediated inhibition during theta oscillation in CA1 pyramidal neurons. Neuroreport 20:1209-1213.

Lakatos P, Gross J, Thut G (2019) A New Unifying Account of the Roles of Neuronal Entrainment. Current Biology 29:R890-R905.

Larkum ME, Waters J, Sakmann B, Helmchen F (2007) Dendritic spikes in apical dendrites of neocortical layer 2/3 pyramidal neurons. J Neurosci 27:8999-9008.

Ledoux E, Brunel N (2011) Dynamics of networks of excitatory and inhibitory neurons in response to time-dependent inputs. Front Comput Neurosci 5:25.

Lefort S, Tomm C, Floyd Sarria J-C, Petersen CCH (2009) The excitatory neuronal network of the $\mathrm{C} 2$ barrel column in mouse primary somatosensory cortex. Neuron 61:301-316.

Leresche N, Lightowler S, Soltesz I, Jassik-Gerschenfeld D, Crunelli V (1991) Low-frequency oscillatory activities intrinsic to rat and cat thalamocortical cells. The Journal of Physiology 441:155-174. 
Llinás R, Jahnsen H (1982) Electrophysiology of mammalian thalamic neurones in vitro. Nature 297:406-408.

Lörincz A, Notomi T, Tamás G, Shigemoto R, Nusser Z (2002) Polarized and compartmentdependent distribution of HCN1 in pyramidal cell dendrites. Nat Neurosci 5:1185-1193.

Lüthi A, McCormick DA (1998) H-current: properties of a neuronal and network pacemaker. Neuron 21:9-12.

Madisen L et al. (2012) A toolbox of Cre-dependent optogenetic transgenic mice for lightinduced activation and silencing. Nat Neurosci 15:793-802.

McCormick DA, Pape HC (1990) Properties of a hyperpolarization-activated cation current and its role in rhythmic oscillation in thalamic relay neurones. The Journal of Physiology 431:291-318.

Muñoz W, Tremblay R, Levenstein D, Rudy B (2017) Layer-specific modulation of neocortical dendritic inhibition during active wakefulness. Science 355:954-959.

Perrenoud Q, Pennartz CMA, Gentet LJ (2016) Membrane Potential Dynamics of Spontaneous and Visually Evoked Gamma Activity in V1 of Awake Mice. PLoS biology 14:e1002383.

Peyrache A, Destexhe A (2019) Electrophysiological monitoring of inhibition in mammalian species, from rodents to humans. Neurobiol Dis 130:104500.

Peyrache A, Seibt J (2020) A mechanism for learning with sleep spindles. Philos Trans R Soc Lond B Biol Sci 375:20190230.

Phillips WA, Larkum ME, Harley CW, Silverstein SM (2016) The effects of arousal on apical amplification and conscious state. Neurosci Conscious 2016:niw015.

Richardson MJE, Brunel N, Hakim V (2003) From Subthreshold to Firing-Rate Resonance. Journal of Neurophysiology 89:2538-2554.

Rossant C, Kadir SN, Goodman DFM, Schulman J, Hunter MLD, Saleem AB, Grosmark A, Belluscio M, Denfield GH, Ecker AS, Tolias AS, Solomon S, Buzski G, Carandini M, Harris KD (2016) Spike sorting for large, dense electrode arrays. Nature Neuroscience 19:634-641.

Schaffer ES, Ostojic S, Abbott LF (2013) A complex-valued firing-rate model that approximates the dynamics of spiking networks. PLoS Comput Biol 9:e1003301.

Schubert D, Kötter R, Staiger JF (2007) Mapping functional connectivity in barrel-related columns reveals layer- and cell type-specific microcircuits. Brain Struct Funct 212:107119. 
Senzai Y, Fernandez-Ruiz A, Buzsáki G (2019) Layer-Specific Physiological Features and Interlaminar Interactions in the Primary Visual Cortex of the Mouse. Neuron 101:500513.e5.

Shriki O, Hansel D, Sompolinsky H (2003) Rate models for conductance-based cortical neuronal networks. Neural Comput 15:1809-1841.

Shu Y, Hasenstaub A, McCormick DA (2003) Turning on and off recurrent balanced cortical activity. Nature 423:288-293.

Sirota A, Montgomery S, Fujisawa S, Isomura Y, Zugaro M, Buzsáki G (2008) Entrainment of neocortical neurons and gamma oscillations by the hippocampal theta rhythm. Neuron 60:683-697.

Sohal VS, Zhang F, Yizhar O, Deisseroth K (2009) Parvalbumin neurons and gamma rhythms enhance cortical circuit performance. Nature 459:698-702.

Soltesz I, Lightowler S, Leresche N, Jassik-Gerschenfeld D, Pollard CE, Crunelli V (1991) Two inward currents and the transformation of low-frequency oscillations of rat and cat thalamocortical cells. The Journal of physiology 441:175-197.

Stark E, Eichler R, Roux L, Fujisawa S, Rotstein HG, Buzsáki G (2013) Inhibition-Induced Theta Resonance in Cortical Circuits. Neuron 80:1263-1276.

Talley EM, Cribbs LL, Lee JH, Daud A, Perez-Reyes E, Bayliss D a (1999) Differential distribution of three members of a gene family encoding low voltage-activated (T-type) calcium channels. The Journal of neuroscience : the official journal of the Society for Neuroscience 19:1895-1911.

Tamás G, Somogyi P, Buhl EH (1998) Differentially interconnected networks of GABAergic interneurons in the visual cortex of the cat. J Neurosci 18:4255-4270.

Tiesinga P, Sejnowski TJ (2009) Cortical enlightenment: are attentional gamma oscillations driven by ING or PING? Neuron 63:727-732.

Tikidji-Hamburyan RA, Martínez JJ, White JA, Canavier CC (2015) Resonant Interneurons Can Increase Robustness of Gamma Oscillations. J Neurosci 35:15682-15695.

Timic Stamenic T, Feseha S, Valdez R, Zhao W, Klawitter J, Todorovic SM (2019) Alterations in Oscillatory Behavior of Central Medial Thalamic Neurons Demonstrate a Key Role of CaV3.1 Isoform of T-Channels During Isoflurane-Induced Anesthesia. Cereb Cortex 29:4679-4696.

Tremblay R, Lee S, Rudy B (2016) GABAergic Interneurons in the Neocortex: From Cellular Properties to Circuits. Neuron 91:260-292.

Ulrich D (2002) Dendritic resonance in rat neocortical pyramidal cells. J Neurophysiol 87:27532759. 
Ulrich D (2014) Subthreshold delta-frequency resonance in thalamic reticular neurons. Eur J Neurosci 40:2600-2607.

Urbain N, Fourcaud-Trocmé N, Laheux S, Salin PA, Gentet LJ (2019) Brain-State-Dependent Modulation of Neuronal Firing and Membrane Potential Dynamics in the Somatosensory Thalamus during Natural Sleep. Cell Reports 26:1443-1457.e5.

van Kerkoerle T, Self MW, Dagnino B, Gariel-Mathis M-A, Poort J, van der Togt C, Roelfsema PR (2014) Alpha and gamma oscillations characterize feedback and feedforward processing in monkey visual cortex. Proc Natl Acad Sci USA 111:14332-14341.

Vecchia D, Beltramo R, Vallone F, Chéreau R, Forli A, Molano-Mazón M, Bawa T, Binini N, Moretti C, Holtmaat A, Panzeri S, Fellin T (2020) Temporal Sharpening of Sensory Responses by Layer V in the Mouse Primary Somatosensory Cortex. Curr Biol 30:15891599.e10.

Vinck M, Bos JJ, Van Mourik-Donga LA, Oplaat KT, Klein GA, Jackson JC, Gentet LJ, Pennartz CMA (2015) Cell-Type and State-Dependent Synchronization among Rodent Somatosensory, Visual, Perirhinal Cortex, and Hippocampus CA1. Front Syst Neurosci 9:187.

Volgushev M, Chauvette S, Mukovski M, Timofeev I (2006) Precise long-range synchronization of activity and silence in neocortical neurons during slow-wave oscillations [corrected]. The Journal of neuroscience : the official journal of the Society for Neuroscience 26:5665-5672.

Wang X-J (2010) Neurophysiological and computational principles of cortical rhythms in cognition. Physiol Rev 90:1195-1268.

Wehr M, Zador AM (2003) Balanced inhibition underlies tuning and sharpens spike timing in auditory cortex. Nature 426:442-446.

Welle CG, Contreras D (2016) Sensory-driven and spontaneous gamma oscillations engage distinct cortical circuitry. Journal of Neurophysiology 115:1821-1835.

Wester JC, Contreras D (2012) Columnar interactions determine horizontal propagation of recurrent network activity in neocortex. J Neurosci 32:5454-5471.

Whittington MA, Traub RD, Kopell N, Ermentrout B, Buhl EH (2000) Inhibition-based rhythms: experimental and mathematical observations on network dynamics. Int J Psychophysiol 38:315-336.

Wilson CJ, Higgs MH, Simmons DV, Morales JC (2018) Oscillations and Spike Entrainment. F1000Res 7.

Xue M, Atallah BV, Scanziani M (2014) Equalizing excitation-inhibition ratios across visual cortical neurons. Nature 511:596-600. 
Zhou C, Liu J, Chen X-D (2012) General anesthesia mediated by effects on ion channels. World J Crit Care Med 1:80-93.

Zucca S, Pasquale V, Lagomarsino de Leon Roig P, Panzeri S, Fellin T (2019) Thalamic Drive of Cortical Parvalbumin-Positive Interneurons during Down States in Anesthetized Mice. Curr Biol 29:1481-1490.e6. 